\title{
DESIGN OF OXYGEN MONITOR AUTOMATIC TEST BASED ON COMMUNICATION PLATFORM
}

\author{
Qiangqiang Xiong ${ }^{1}$, ke Li ${ }^{1}$, Xiaojuan Fang ${ }^{2}$, Meilin Zeng ${ }^{2}$, Jianpeng Liu' ${ }^{1}$, Muhammad Aqeel Ashraf ${ }^{4}$, Wenlei Xu ${ }^{5}$ \\ ${ }^{1}$ Nanchang Institute of Technology, Nanchang, China \\ 2Jiangxi University of Finance and Economics, Jiangxi, China \\ 3 Jiangxi Vocational Technical college of industry Trade, Jiangxi, China \\ ${ }^{4}$ Department of Environmental Science \& Engineering, China University of Geosciences, Wuhan 430074, China \\ ${ }^{5}$ Nanchang Danxun Electronic Technology Co., Ltd., Nanchang, China
}

\begin{abstract}
Airborne oxygen monitor is an airborne equipment to regulate and monitor pilots' breathing supply. In order to improve the adaptability of communication interface, this paper studies the bus interface technology based on single signal line, and designs and improves a new single-bus interface scheme suitable for the current application environment. The interface protocol is to take a single signal line, and design the corresponding host interface circuit and the machine interface circuit. It realize that conversion between serial port of MSP430 single chip computer and USB port via PL2303 chip. It is found that the oxygen monitor automatic test platform can detect the oxygen monitor in real time and calibrate it automatically. The experimental results show that, when the transmission medium is selected as no. 5 twisted pair, the communication distance of the new bus can reach $200 \mathrm{~m}$, the transmission rate can reach $38400 \mathrm{bit} / \mathrm{ms}$, and the number of slave nodes can be driven up to 450 . The bus performs well in both distance and node drive.
\end{abstract}

Keywords: Automatic Test Communication Platform, Oxygen Monitor, Design.

\section{Introduction}

Airborne oxygen monitor, as an important part of airborne oxygen system, is an indispensable airborne electronic equipment to ensure normal breathing of pilots. When the oxygen produced by the system cannot meet the requirements of aviation oxygen, the oxygen monitor will make alarm, automatically or manually prompt the pilot to switch to the emergency oxygen source, to ensure the safety of the pilot's life.

The oxygen monitor mainly detects the oxygen partial pressure of the pilot's breathing air source in real time, and reminds the pilot of the abnormal oxygen supply in the way of alarm, so as to ensure the normal oxygen supply of the aircraft at different altitudes ${ }^{[1]}$. The oxygen monitor has built-in oxygen partial pressure sensor, and is connected with the air source that the pilot breathes. The oxygen partial pressure measured by the oxygen partial pressure sensor is one of the core parameters of the oxygen monitor, which is the internal judgment basis of the monitor alarm [2].

Research on Luo M shows that Bus USES masterslave serial asynchronous communication, which requires communication interfaces to distinguish the host from the slave. Although the part from the machine has a transceiver chip TSS721A terminal developed by TI company as the core from machine parts processing, but in the host has applicationspecific integrated circuit part is not yet available, so every company and factory according to the M - Bus standard designed circuit 1151 at present, domestic or foreign research application of single-bus technology limited to low speed and small power consumption situation ${ }^{[3]}$.A single data line bi directional dual voltage communication interface circuit is presented in Hong F.

This interface circuit uses a single data line to realize two-way reading and writing of data in a time-sharing way, and different high level voltages are used for sending and receiving, which is especially suitable for networking communication of passive low-power modules ${ }^{[4]}$.Yao J Y proposed a non-polar communication interface for a wide voltage single data line. The interface circuit can realize single data line non-polarity serial power consumption, send and receive signal voltage range wide, can improve the adaptability of communication interface. However, the above communication interfaces still need to be improved in multi-node communication applications ${ }^{[5]}$.

The innovation of this paper is that the communication interface scheme adopts the singlesignal line master-slave structure, designs the corresponding host interface circuit and slave interface circuit, designs a portable communication calibration module, and applies it to the oxygen 
monitor automation test platform. This extends the debugging method of this type of oxygen monitor.

\section{System Design Method}

\subsection{Hardware design of oxygen monitor automatic test platform communication calibration module}

In the process of testing a series of airborne oxygen monitor, the main function of the test is mainly achieved by the serial communication of the oxygen monitor. Whether it is the oxygen monitor debugging calibration or normal operation of the data output, are inseparable from the oxygen monitor communication interface reading and writing.

According to its special working environment and mode, the oxygen monitor adopts a communication interface compatible with the bus interface described in this paper, it is configured with a simple and applicable communication protocol to realize single-to-single data communication ${ }^{[6]}$.

\subsubsection{The communication interface of the oxygen monitor}

The oxygen monitor adopts C8051 series singlechip microcomputer chip as the control core, and is equipped with a new type of bus from the computer side hardware interface circuit, in order to ensure the stability and reliability of data transmission, and also to facilitate the connection with external equipment, as shown in figure 1.

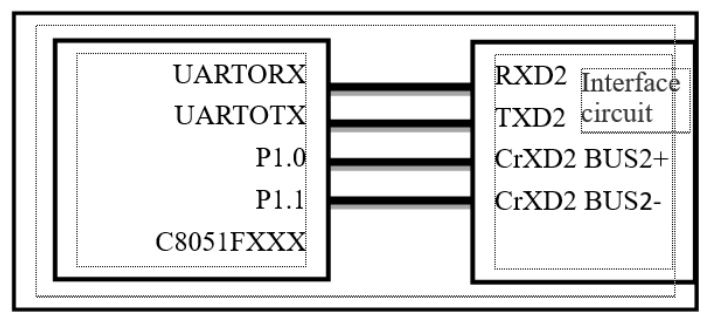

Figure 1: Interface connection diagram of a certain type of oxygen monitor

\subsubsection{Oxygen monitor automated test platform communication interface}

Oxygen monitor automation test platform is one kind which based on industrial computer automatic test system for airborne oxygen monitor, accurately and efficiently simulate aircraft negative pressure environment, onboard oxygen generating system, extreme power supply condition and the drive motor of variable load, supplemented by high precision flow rate, pressure, speed and torque sensor to achieve comprehensive performance testing oxygen monitor ${ }^{[7-8]}$.

The communication between the oxygen monitor automated test platform and the oxygen monitor is carried out through the oxygen monitor automated test platform communication calibration module.

As shown in figure 2, it is the hardware architecture diagram of this communication module, which not only completes the communication conversion between the oxygen monitor and the ipc, but also provides the function of online debugging and calibration of the oxygen monitor on an ordinary computer.

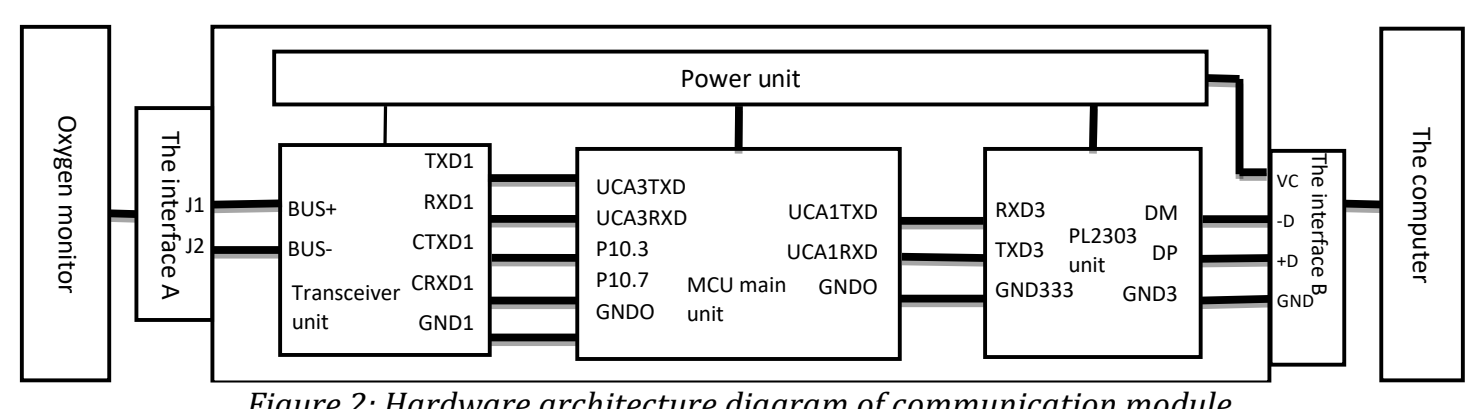

Figure 2: Hardware architecture diagram of communication module

The whole communication calibration module consists of four parts, which are MCU main control unit, power generation road unit, PL203 unit and power unit.

\subsection{Communication protocols and data formats}

The communication calibration module carries out serial communication with the oxygen monitor through the UCA3 serial port, and the baud rate of communication is $1200 \mathrm{bit} / \mathrm{s}$. The communication mode adopts the mode of sending and receiving single-byte data in time sharing, and the data packet composed of multi-bytes is one frame.

\subsection{The software design of oxygen monitor automatic test platform communication module}

\subsubsection{Main program flow}

MSP430F5438 single-chip microcomputer as a Flash single-chip, can be through the JTAG port on the single-chip rapid, large capacity, programming in the system. The IRA integrated development environment is used to program and debug the MCU.

The system automatically enters low power mode to reduce power consumption when there is 
no data transmission. The two hardware interrupts UCA3 and UCA1 receive data from both ends, and the interrupts from the computer UCA1 have a higher priority than the interrupts from the oxygen monitor UCA3. According to the running characteristics of the oxygen monitor, the communication module contains two working modes:

1. Acquisition mode: in this mode, the oxygen monitor continuously updates the current oxygen partial pressure data to the ipc through the communication calibration module;

2. Debug mode: in this mode, the ipc calibrates and debugs the oxygen monitor through the communication calibration module.

\subsubsection{The initialize}

There are four serial communication modes of MSP430F5438 MCU, namely UART mode, line mode, address mode and baud rate detection mode, which are controlled and selected by UCMODE. In the communication module described in this paper, UART mode is adopted by both communication terminals. This is the most commonly adopted working mode and the simplest one ${ }^{[9]}$.

\subsubsection{To send and receive function}

In this paper, the realization of MCU serial port communication function mainly depends on the corresponding transceiver function, which are the transceiver function of ipc and the transceiver function of the device under test.UCA3, which communicates with the oxygen monitor, is in the receiving state by default. By receiving the interrupt, the MCU can continuously collect the data from the oxygen monitor, and judge the first byte is saved as four consecutive bytes of the characteristic value Oxfi, which are regarded as useful data, and the rest are discarded. There are use finish at the same time, each receiving a set of data, MCU will through setting flags, notice the background process ${ }^{[10]}$.

\section{Results Analysis}

\subsection{Hardware debugging results analysis}

After the hardware circuit board is welded, it needs to be inspected. The main way of inspection is to test the voltage of several key points by oscilloscope. The first is the detection of the power module, power output including $3.3 \mathrm{~V}, 12 \mathrm{~V}$ and $20 \mathrm{~V}$ ensure the stability of power supply voltage output.

Then observe whether the base or gate voltage of the triode or MOS tube is within the rated range of the device.

Finally, the output voltage detection, voltage output amplitude and whether there will be 'burr, overshoot and other phenomena. In the debugging process, not only the function of the signal input port needs to be detected, but also the function of the control port needs to be detected to observe whether the switch state of the circuit is normal.

Table 1: Signal propagation delay (us) under different transmission conditions during data downlink

\begin{tabular}{|c|c|c|c|c|}
\hline \multirow{2}{*}{$\begin{array}{c}\text { Transmission } \\
\text { rate } \\
\text { (bit/s })\end{array}$} & 500 & 100 & 1500 & 2000 \\
\cline { 2 - 5 } & & & & \\
\hline 1200 & $4.29 / 3.96$ & $7.34 / 5.83$ & $10.44 / 7.08$ & $13.37 / 8.67$ \\
\hline 4800 & $4.53 / 4.11$ & $7.41 / 6.04$ & $10.36 / 7.06$ & $13.45 / 8.62$ \\
\hline 9600 & $4.41 / 4.01$ & $7.39 / 6.16$ & $10.98 / 8.51$ & $13.96 / 8.52$ \\
\hline 19200 & $4.39 / 3.93$ & $7.85 / 5.97$ & $10.58 / 8.67$ & $13.86 / 9.14$ \\
\hline 38400 & $4.53 / 4.05$ & $8.06 / 6.35$ & $10.46 / 8.20$ & $13.95 / 9.39$ \\
\hline
\end{tabular}

The higher the transmission rate of the signal, the higher the requirement of symmetry between the rising propagation delay and the falling propagation delay.

As can be seen from table 1, with the shortening of transmission distance, the downlink signal propagation delay will be shorter and the symmetry will be better.

Therefore, in order to obtain a higher transmission rate, the new bus interface can be realized by shortening the transmission distance.

\subsection{Analysis of software debugging results}

After the completion of hardware debugging and testing to ensure that the peripheral circuit can work reliably, the communication calibration module will be further debugged in software. The debugged object is the control chip of the communication calibration module.

The main work is to download the program, and online debugging, know to meet the basic functional requirements, download and debugging software as described above, using the IAR embedded IDE, its debugging interface as shown in the figure below.

Firstly, connect the circuit board to the power supply, connect the computer to the circuit board JTAG through a special debugging adapter, download the written communication calibration program to the board, set the breakpoint, run the program, and observe the value of key registers. 
Table 2: Receives the oxygen monitor data frames

\begin{tabular}{|c|c|c|}
\hline $\begin{array}{c}\text { Characterist } \\
\text { ics of bytes }\end{array}$ & $\begin{array}{c}\text { Oxygen partial } \\
\text { pressure data }\end{array}$ & $\begin{array}{c}\text { Characteristics } \\
\text { of the logo }\end{array}$ \\
\hline OxF3 & BYTE1, BYTE2 & BYTE3 \\
\hline
\end{tabular}

The received data is an information frame composed of four bytes, with BYTE0 as the characteristic byte. In order to determine the value OxF3, the communication calibration module starts by identifying this as the data frame. BYTE1 and BYTE2 are data bytes, providing the oxygen partial pressure data at that time; BYTE3 is the characteristic flag byte that indicates the receiving state in the previous loop.

Table 3: Data frames sent to the oxygen monitor

\begin{tabular}{|c|c|c|}
\hline $\begin{array}{c}\text { Characteristics } \\
\text { of bytes }\end{array}$ & To send data & $\begin{array}{c}\text { Check } \\
\text { byte }\end{array}$ \\
\hline BYTE0 & BYTE1, BYTE2 & BYTE3 \\
\hline
\end{tabular}

The data sent is also a data frame composed of four bytes. BYTE0 is the characteristic byte and command byte, indicating the operation to be carried out. BYTE1 and BYTE2 are data bytes, providing the calibration constant; BYTE3 is the check byte to ensure the correctness of the transmission.

\subsection{System test result analysis}

After the completion of hardware debugging and software debugging, the communication calibration module will be connected to the oxygen monitor automated test platform, and the final overall test will be carried out. If the oxygen partial pressure value is combined with the simulated state at that time, there will be a standard range, as shown in the "standard range" column below.

When the oxygen partial pressure value is not within the standard range, it indicates that there is a fault in the oxygen monitor, and the oxygen monitor needs to be calibrated, that is, the oxygen monitor automated test platform needs to carry out information interaction with the oxygen monitor. Click the "oxygen partial pressure calibration" button and the "oxygen monitor" window will pop up to select zero point calibration, linear calibration or binary integral calibration. The function of the oxygen monitor communication calibration module can be directly reflected in the process of software operation. The requirements for oxygen monitor read-write functionality are included in the test coverage of the oxygen monitor automation test platform. Click the "test report" button to generate the oxygen monitor test report, and then click the "open report" button to open the oxygen monitor test record table as shown in the figure.

As shown in the dotted box, is the test result of the oxygen monitor communication calibration function.

\section{Conclusion}

Oxygen monitor automation platform bus interface circuit has the characteristics of single signal line, high drive, non-polarity, low power consumption, and simple structure, low cost. It is also suitable for serial communication of external devices such as sensors and meters. After analyzing the transmission line theory, the new bus interface and the transmission line were modeled and simulated using Multisim software, and it was found that the new bus interface performed well in both distance and node drive.

\section{References}

[1] Liu C T, Hong-Cai L I, Zhen-Xin H E, et al, "Design of Fiber Bragg Grating Automatic Analysis Test System Based on LabVIEW[J]", Acta Photonica Sinica, 2016, Volume 45, Issue 2, Pages 206002.

[2] Zhang W, "Design of Radio Frequency Link in Automatic Test System for Multimode Mobile Communication Base Station [J]", International Journal of Bifurcation \& Chaos, 2016, Volume 25, Issue 14, Pages 1540032.

[3] Luo M, Peng Z, Li J, "Design of a four-channel automatic test system for servos[C]", Guidance, Navigation \& Control Conference, 2017.

[4] Hong F, Zuo L, Jiang Y, et al, "Automatic test system of transformer protection design in intelligent substation [C]", International Conference on Renewable Power Generation, 2016.

[5] Yao J Y, Chao D, "Design of fuel valve automatic performance test system based on LabVIEW [C]", IEEE International Conference on Industrial Engineering \& Engineering Management, 2016.

[6] Haydar 0, Semih K 0, "A Novel Automatic Rapid Diagnostic Test Reader Platform [J]", Computational \& Mathematical Methods in Medicine, 2016, Issue 6, Pages 1-10.

[7] Mani P, Prasanna M, "Automatic test case generation for programmable logic controller using function block diagram [C]", International Conference on Information Communication \& Embedded Systems, 2016.

[8] Alizadeh B, Sharafinejad S R, "Incremental SATbased Accurate Auto-correction of Sequential Circuits through Automatic Test Pattern Generation [J]", IEEE Transactions on ComputerAided Design of Integrated Circuits and Systems, 2018, Issue 99, Pages 1-1.

[9] Xiong B, Jiang L, Yang L, et al, “Design and test of electromechanical control system of automatic feeder for dairy cow [J]. Transactions of the Chinese Society of Agricultural Engineering, 2017, Volume 33, Issue 7, Pages 157-163.

[10] Saranya C, Sreedevi S, Jose S, et al, "Design of inter process communication methodology in automatic spacecraft checkout environment [C]", India Conference, 2017. 